Article

\title{
Analytical Estimates of Critical Taylor Number for Motion between Rotating Coaxial Cylinders Based on Theory of Stochastic Equations and Equivalence of Measures
}

\author{
Artur V. Dmitrenko 1,2
}

check for updates

Citation: Dmitrenko, A.V. Analytical Estimates of Critical Taylor Number for Motion between Rotating Coaxial Cylinders Based on Theory of Stochastic Equations and Equivalence of Measures. Fluids 2021, 6, 306. https://doi.org/10.3390/fluids6090306

Academic Editor: Laura A. Miller

Received: 30 June 2021

Accepted: 27 August 2021

Published: 30 August 2021

Publisher's Note: MDPI stays neutral with regard to jurisdictional claims in published maps and institutional affiliations.

Copyright: (C) 2021 by the author. Licensee MDPI, Basel, Switzerland. This article is an open access article distributed under the terms and conditions of the Creative Commons Attribution (CC BY) license (https:// creativecommons.org/licenses/by/ $4.0 /)$.
1 Department of Thermal Physics, National Research Nuclear University "MEPhI", Kashirskoyeshosse 31, 115409 Moscow, Russia; AVDmitrenko@mephi.ru

2 Department of Thermal Engineering, Russian University of Transport "MIIT", Obraztsova Street 9, 127994 Moscow, Russia

\begin{abstract}
The purpose of this article was to present the solution for the critical Taylor number in the case of the motion between rotating coaxial cylinders based on the theory of stochastic equations of continuum laws and the equivalence of measures between random and deterministic motions. Analytical solutions are currently of special value, as the solutions obtained by modern numerical methods require verification. At present, in the scientific literature, there are no mathematical relationships connecting the critical Taylor number with the parameters of the initial disturbances in the flow. The result of the solution shows a satisfactory correspondence of the obtained analytical dependence for the critical Taylor number to the experimental data.
\end{abstract}

Keywords: stochastic equations; equivalence of measures; nature of turbulence; critical Reynolds number

\section{Introduction}

Analytical solutions are currently of special value, as the solutions obtained by modern numerical methods require verification. As it is known, an advantage of analytical formulas is the visualization of physical relationships between quantities. Therefore, the development of physical and mathematical theories for complex physical nonlinear processes, which are described by inhomogeneous high-order partial differential equations, is especially significant.

Moreover, analytical dependences including theoretical estimates are extremely important in the analysis of experimental data, when it is necessary to take into account the effect of substantial quantities, which are random in time and space, instead of only to give average statistical estimates.

Different ideas of the theory of turbulence are presented in [1-10]. Mathematical methods for obtaining solutions of the Navier-Stokes equation, the theory of solitons, and the theory of strange attractors are presented in [11-24].

Numerical DNS methods, and stochastic and statistical equations for investigating turbulent motion are given in [25-34]. Special attention was given to the theoretical solutions for the critical Reynolds number. It should be noted that the most well-known ratio based on the theory of dimension was determined using experimental data [35-40]. Therefore, based on these experimental formulas, it was impossible to obtain a new theory for determining analytical dependences for the critical Reynolds number of turbulence in different flows.

The theory of turbulence based on stochastic equations and the theory of equivalent measures makes it possible to derive analytical dependences for the first and second critical Reynolds numbers in the cases of isothermal and nonisothermal flows on a smooth flat plate and in a round tube [41-44]. The progress of this theory gives a new method for determining 
analytical dependences for profiles of averaged velocity and temperature fields [45-47], the friction and heat-transfer coefficients [48-50], second-order correlations [51,52], the correlation dimension of an attractor in the boundary layer [53-56], and the formulas for the Reynolds analogy [56-58] in theoretical solutions for spectral functions of the turbulent medium [59-63].

As a result, it was determined that the spectrum $E(k)_{j}$ depends on wave number $k$ for the interval of the generation of turbulence in the form of $E(k)_{j} \sim k^{n}, n=-1.2 /-1.5,-1.66<n<-1$. This formula was named as the ratio of uncertainty in turbulence generation $[62,63]$.

Other types of fluid motions, which are important for both theory and practice, were also investigated. For example, the determination of the critical Reynolds number in a jet and for the flow near a rotating disk is presented in $[64,65]$.

Thus, we presented here the analytical solution for the critical Taylor number in the case of the motion between rotating coaxial cylinders.

\section{Equations of Conservation for the Stochastic Process}

The equations were derived in [39-41] and take the following form:

The equation of mass (continuity)

$$
\frac{d(\rho)_{c o l s t}}{d \tau}=-\frac{(\rho)_{s t}}{\tau_{c o r}}-\frac{d(\rho)_{s t}}{d \tau}
$$

the momentum equation

$$
\frac{d(\rho \vec{U})_{c o l s t}}{d \tau}=\operatorname{div}\left(\tau_{i, j}\right)_{c o l s t}+\operatorname{div}\left(\tau_{i, j}\right)_{s t}-\frac{(\rho \vec{U})_{s t}}{\tau_{c o r}}-\frac{d(\rho \vec{U})_{s t}}{d \tau}+F_{c o l s t}+F_{s t}
$$

and the energy equation

$$
\frac{d E_{c o l s t}}{d \tau}=\operatorname{div}\left(\lambda \frac{\partial T}{\partial x_{j}}+u_{i} \tau_{i, j}\right)_{c o l s t}+\operatorname{div}\left(\lambda \frac{\partial T}{\partial x_{j}}+u_{i} \tau_{i, j}\right)_{s t}-\left(\frac{E_{s t}}{\tau_{c o r}}\right)-\left(\frac{d E_{s t}}{d \tau}\right)+\left(u_{i} F\right)_{c o l s t}+\left(u_{i} F\right)_{s t}
$$

Here, $E, \rho, \vec{U}, u_{i}, u_{j}, u_{l}, \mu, \tau, \tau_{i, j}$ are the energy; the density; the velocity vector; the velocity components in the directions $x_{i}, x_{j}$, and $x_{l}(i, j, l=1,2,3)$, respectively; the dynamic viscosity; the time; and the stress tensor $\tau_{i, j}=P+\sigma_{i, j}$, where $\sigma_{i, j}=\mu\left(\frac{\partial u_{i}}{\partial x_{j}}+\frac{\partial u_{j}}{\partial x_{i}}\right)-$ $\delta_{i j}\left(\xi-\frac{2}{3} \mu\right) \frac{\partial u_{l}}{\partial x_{l}}, i$ and $j$ are the tensorial notations, $\delta_{i j}=1$ if $I=j, \delta_{i j}=0$ for $i \neq j . j$ is the pressure of a liquid or gas; $\lambda$ is the thermal conductivity; $c_{p}$ and $c_{v}$ are the specific heat at constant pressure and volume, respectively; $F$ is the external force. Further, $L=L_{U, P}=L_{U}$ is the scale of turbulence. The subscripts $(\mathrm{U}, \mathrm{P})$ and $(\mathrm{U})$ refer to the velocity field and the subscript ${ }_{\mathrm{T}}$ ) refers to the temperature field. The turbulence scale $L$ is taken along the radius $r$. The subscript "col st" refers to the components that are deterministic. The subscript "st" refers to the components that are stochastic. Then, for the nonisothermal motion of the medium, using the definition of equivalence of measures between deterministic and random processes at the critical point, the sets of stochastic equations of energy, momentum, and mass are defined for the next space-time areas: (1) the onset of generation (subscript $1,0$ or 1$)$; (2) the generation (subscript 1,1$)$; (3) the diffusion $(1,1,1)$; and (4) the dissipation of the turbulent fields.

\section{Stochastic Equations for Critical Taylor Number}

For the critical numbers, sets (1)-(3) of the equations of mass, momentum, and energy for the area $(1)$ referring to the pair $(N, M)=(1,0)$ is:

$$
\left(\frac{d(\rho)_{c o l, s t}}{d \tau}\right)_{1,0}=-\frac{\rho_{s t}}{\tau_{c o r}}
$$




$$
\begin{gathered}
\left\{\begin{array}{l}
\left(\frac{d(\rho \vec{U})_{c o l, s t}}{d \tau}\right)_{1,0}=-\left(\frac{(\rho \vec{U})_{s t}}{\tau_{c o r}}\right) ; \\
\operatorname{div}\left(\tau_{i, j}\right)_{c o l, s t 1}=\frac{(\rho \vec{U})_{s t}}{\tau_{c o r}},
\end{array}\right. \\
\left\{\begin{array}{l}
\left(\frac{d(E)_{c o l s t}}{d \tau}\right)_{1,0}=-\left(\frac{(E)_{s t}}{\tau_{c o r}}\right)_{1,0^{\prime}} \\
\operatorname{div}\left(\lambda \frac{\partial T}{\partial x_{j}}+u_{i} \tau_{i, j}\right)_{c o l, s t 1}=\left(\frac{(E)_{s t}}{\tau_{c o r}}\right)_{1,0}
\end{array} .\right.
\end{gathered}
$$

The motion between rotating coaxial cylinders is described in detail in [34-38]. In particular, for the laminar motion during the rotation of both cylinders, the velocity profile is determined for laminar motion by the dependence [31]

$$
u_{r}=\left[\frac{1}{r_{2}^{2}-r_{1}^{2}}\right]\left[r\left(\omega_{2} r_{2}{ }_{2}-\omega_{1} r_{1}^{2}\right)-\frac{r^{2}{ }_{2}{ }^{2}}{r}\left(\omega_{2}-\omega_{1}\right)\right] .
$$

In the case when the inner cylinder rotates and the outer cylinder is at rest, it is also characterized by the bifurcation in laminar motion with the formation of Taylor vortices instead of only by the transition of deterministic motion to the random motion. Then, the velocity profile for the laminar motion without the formation of Taylor vortices [31] is

$$
u_{r}=\left[\frac{1}{d\left(r_{2}+r_{1}\right)}\right]\left[\frac{r^{2}{ }_{2} r_{1}}{r}\left(\omega_{1}\right)-r\left(\omega_{1} r_{1}{ }_{1}\right)\right] .
$$

In Formulas (5) and (6), $r_{1}, r_{2}, \omega_{1}, \omega_{2}$, and $d$ are the radius and speed of rotation of the first and second cylinders, and the distance between the cylinders, respectively.

Taking into account the set of stochastic equations of the presented theory of equivalent measures for a continuous isothermal medium, we write for the space-time region of the beginning of the resonance-correlation "determinism-randomness" according to the equivalence of measures (4) of deterministic and random motion, and we define the derivative

$$
\begin{gathered}
\frac{\partial u_{r}}{\partial r}=\frac{\partial}{\partial r}\left[\frac{\omega_{1}\left(r_{1}^{2}\right)}{d\left(r_{2}+r_{1}\right)}\right]\left[\frac{r_{2}^{2}}{r}-r\right]=\left[\frac{\omega_{1}\left(r_{1}^{2}\right)}{d\left(r_{2}+r_{1}\right)}\right]\left[-\frac{r^{2} 2}{r^{2}}-1\right] \\
\frac{\partial u_{r}}{\partial r}=\frac{\partial}{\partial r}\left[\frac{\omega_{1}\left(r_{1}^{2}\right)}{d\left(r_{2}+r_{1}\right)}\right]\left[\frac{r_{2}^{2}}{r}-r\right]=\left[\frac{\omega_{1}\left(r_{1}^{2}\right)}{d\left(r_{2}+r_{1}\right)}\right]\left[-\frac{r_{2}^{2}}{r^{2}}-1\right] \\
\mu\left(\frac{\partial u_{r}}{\partial r}\right)^{2}=\rho \cdot v \cdot\left[\frac{\omega_{1}\left(r_{1}{ }_{1}\right)}{d\left(r_{2}+r_{1}\right)}\right]^{2}\left[\frac{r_{2}^{2}}{r^{2}}+1\right]^{2}=\rho \cdot v \cdot\left[\frac{\omega_{1}}{d}\right]^{2}\left[\frac{\left(r^{2}{ }_{1}\right)}{\left(r_{2}+r_{1}\right)}\right]^{2}\left[\frac{r^{2}{ }_{2}+r^{2}}{\left(r^{2}\right)}\right]^{2}
\end{gathered}
$$

Transforming Equation (7), we obtain

$$
\mu\left(\frac{\partial u_{r}}{\partial r}\right)^{2}=\rho \cdot v \cdot \omega_{1}^{2}\left[\frac{\left(r_{1}^{4}\right)}{d^{2}}\right]\left[\frac{1}{r_{1}\left(2+d / r_{1}\right)}\right]^{2}\left[\frac{r^{2}{ }_{2}+r^{2}}{\left(r^{2}\right)}\right]^{2} \approx \rho \cdot v \cdot \omega_{1}{ }^{2}\left[\frac{\left(r^{2}{ }_{1}\right)}{d^{2}}\right] .
$$

then

$$
\mu\left(\frac{\partial u_{r}}{\partial r}\right)^{2} \approx \rho \cdot v \cdot \omega_{1}^{2}\left[\frac{\left(r^{2}\right)}{d^{2}}\right]=\left|\frac{E_{s t}}{\tau_{c o r}^{0}}\right|_{1,0}
$$

or we have $\left(\frac{v}{d^{2}} \tau_{c o r}^{0}\right)\left(\frac{r^{2} \omega_{1}^{2}}{E_{s t} / \rho}\right)=1$. 
For the case of the time correlation $\left(\tau_{c o r}^{0}\right)_{1,0}=\frac{L}{\sqrt{E_{s t} / \rho}}$, we can write

$$
\left(\frac{v}{d^{2}} \frac{L}{\sqrt{E_{s t} / \rho}}\right)\left(\frac{r^{2}{ }_{1} \omega_{1}^{2}}{E_{s t} / \rho}\right)=1
$$

or

$$
\left(\left(\frac{v}{r_{1} \omega_{1} d}\right) \frac{L}{d}\right)\left(\frac{r_{1} \omega_{1}}{\sqrt{E_{s t} / \rho}}\right)^{3}=1
$$

Thus, we write that

$$
\left(\frac{r_{1} \omega_{1} d}{v}\right)=\left(\frac{r_{1}}{d} \frac{L}{r_{1}}\right)\left(\frac{r_{1} \omega_{1}}{\sqrt{E_{s t} / \rho}}\right)^{3}
$$

and obtain the dependence

$$
\mathrm{Ta}=\left(\frac{r_{1} \omega_{1} d}{v}\right) \sqrt{\frac{d}{r_{1}}}=\left(\sqrt{\frac{r_{1}}{d}}\left(\frac{L}{r_{1}}\right)\right)\left(\frac{r_{1} \omega_{1}}{\sqrt{E_{s t} / \rho}}\right)^{3}
$$

and, finally, the formula for the critical Taylor's number is

$$
\mathrm{Ta}=\left(\frac{r_{1} \omega_{1} d}{v}\right) \sqrt{\frac{d}{r_{1}}}=\left(\sqrt{\frac{r_{1}}{d}}\left(\frac{L}{d}\right)\left(\frac{d}{r_{1}}\right)\right)\left(\frac{r_{1} \omega_{1}}{\sqrt{E_{s t} / \rho}}\right)^{3}=\left(\sqrt{\left(\frac{d}{r_{1}}\right)}\right)\left(\frac{r_{1} \omega_{1}}{\sqrt{E_{s t} / \rho}}\right)^{3}\left(\frac{L}{d}\right)
$$

\section{Critical Point in the Case of Motion between Rotating Coaxial Cylinders}

We determine the position of the critical point. The critical point definition is found from the equation

$$
\int_{-\Delta V \mid 2}^{+\Delta V \mid 2} d\left(E_{c o l_{s t}}\right)_{1 ; 0}=\int_{X} d E_{s t}
$$

$E_{s t}$ is the random energy component in the space $X$ with the measure $m\left(E_{s t}\right)<\infty$

$$
E_{s t}=E_{s t}\left(\vec{x}_{i}, \tau_{i}, m_{i}\right)<\infty
$$

In accordance with ergodic theory,

$$
\int_{X} d E_{s t}=\frac{1}{\Delta V} \int_{V} E_{s t} \delta\left((\Delta V)_{\text {critic }}-\Delta V\right) d V=\frac{1}{\tau_{c o r}^{0}} \int_{\tau} E_{s t} \delta\left(\tau_{\text {cor }}^{0}-\tau\right) d \tau=\left(E_{s t}\right)_{\text {critic }}
$$

$\left(E_{s t}\right)_{c r i t i c}$ is the energy of the stochastic field at the critical point, or

$$
\int_{X} d E_{s t}=\frac{1}{L} \int_{L} E_{s t} \delta\left(\left(x_{i}\right)_{\text {critic }}-x_{i}\right) d L=\frac{1}{\tau_{c o r}^{0}} \int_{\tau} E_{s t} \delta\left(\tau_{c o r}^{0}-\tau\right) d \tau=\left(E_{s t}\right)_{\text {critic }}
$$

$L$ is the scale of disturbance. Then, we can write

$$
\begin{gathered}
\int_{-V \mid 2}^{+V \mid 2} d\left(E_{c o l_{s t}}\right)_{1 ; 0} \cong\left(0,5 \rho u_{r}^{2}\right)_{-L / 2}^{+L / 2}=0,5 \rho \omega_{1}^{2}\left(\left[\frac{\left(r^{2}\right)}{d\left(r_{2}+r_{1}\right)}\right]\left[\frac{r_{2}^{2}}{r}-r\right]\right)_{-L / 2}^{2+L / 2}= \\
=0.5 \rho \omega^{2}\left[\frac{r^{2}{ }_{1}}{d\left(r_{2}+r_{1}\right)}\right]^{2}\left\{\left[\frac{r_{2}{ }_{2}}{(r+L / 2)}-(r+L / 2)\right]^{2}-\left[\frac{r_{2}{ }^{2}}{(r-L / 2)}-(r-L / 2)\right]^{2}\right\} \approx 0.5 \rho \omega^{2}\left[\frac{r^{2}{ }_{1}}{d\left(r_{2}+r_{1}\right)}\right]^{2} \frac{3.75 d^{2} r^{2} 2 r L}{(r+L / 2)^{2}(r-L / 2)^{2}}
\end{gathered}
$$


An estimate of Equation (18), when $r \rightarrow r_{1}$ and $r_{2} \geq r_{1}>>d$, may be written as

$$
\begin{aligned}
0.5 \rho \omega_{1}{ }^{2}\left[\frac{r^{2}{ }_{1}}{d\left(r_{2}+r_{1}\right)}\right]^{2} \frac{3.75 d^{2} r_{2}{ }_{2} 2 r L}{(r+L / 2)^{2}(r-L / 2)^{2}}= & 0.5 \rho \omega_{1}{ }^{2}\left[\frac{r^{2}{ }_{1}}{2 d r_{2}}\right]^{2} \frac{3.75 d^{2} r^{2}{ }_{2} 2 r L}{(r+L / 2)^{2}(r-L / 2)^{2}} \approx K \cdot \rho\left(\omega_{1}{ }^{2} r_{1}^{2}\right) \frac{L}{r} \\
\text { Here, } K=0.94 / 0.93, \text { and finally, } & \\
& \int_{-V \mid 2}^{+V \mid 2} d\left(E_{c o l} l_{s t}\right)_{1 ; 0} \approx K \rho \omega_{1}{ }^{2} r^{2}{ }_{1}\left(\frac{L}{d}\right)\left(\frac{d}{r_{1}}\right) \frac{r_{1}}{r}
\end{aligned}
$$

Therefore, we obtain

$$
K \rho \omega_{1}^{2} r^{2}{ }_{1}\left(\frac{L}{d}\right)\left(\frac{d}{r_{1}}\right) \frac{r_{1}}{r} \approx E_{s t}
$$

Then, we finally have the formula for the critical point in the case of the motion between rotating coaxial cylinders

$$
r_{c r} \approx K\left(\frac{d}{r_{1}}\right)\left(\frac{r_{1} \omega_{1}}{\sqrt{E_{s t} / \rho}}\right)^{2}\left(\frac{L}{d}\right) r_{1}
$$

\section{Results of Estimates of the Critical Taylor Number}

We present the results of calculations for the conditions of the experiment of Taylor [31], when $\left(d / r_{1}\right)=0.028$, the pulsation intensity $\left(\frac{\sqrt{E_{s t} / \rho}}{r_{1} \omega_{1}}\right)$ is $1-2 \%$, the relative magnitude of the turbulence scale $(L / d)$ is $0.01-0.02$, and the experimental value of the critical Taylor number is (Ta)cr $400[31,66]$.

We emphasize that it is of interest to make a similar estimate, when the laminarmotion velocity profile is determined by the motion of Taylor vortices, but this version of the definition is not used here.

Thus, the critical Taylor number and critical point using Formulas (13) and (22), and depending on the values of the pulsation intensity and the scale determined from experiments, may be calculated as

$$
\begin{gathered}
T a=\left(\sqrt{\left(\frac{d}{r_{1}}\right)}\right)\left(\frac{r_{1} \omega_{1}}{\sqrt{E_{s t} / \rho}}\right)^{3}\left(\frac{L}{d}\right)=(\sqrt{(0.028)})(62 .)^{3}(0.01)=398 \\
r_{c r} \approx 0.93\left(\frac{d}{r_{1}}\right)\left(\frac{r_{1} \omega_{1}}{\sqrt{E_{s t} / \rho}}\right)^{2}\left(\frac{L}{d}\right) r_{1}=0.93(62 .)^{2}(0.01)(0.028) r_{1}=1.0009 r_{1}
\end{gathered}
$$

The accuracy and the physical validity of the expression for the critical point can be verified indirectly. Thus, having experimental relationships for quantities $\left(\frac{d}{r_{1}}\right),\left(\frac{L}{d}\right)$, it is possible to obtain an indirect empirical value for the scale of turbulence. At the same time, calculating the distance from the surface of the first cylinder to the critical point $\Delta r=r_{c r}-r_{1}$, it is necessary to take the ratio of the scale to this distance. The resulting value must be compared with the von Karman constant.

Thus, $\left(\frac{d}{r_{1}}\right)=0.028,\left(\frac{L}{d}\right)=0.01$; thus, $L=0.00028 r_{1}$. The distance from the surface of the first cylinder to the critical point is $\Delta r=r_{c r}-r_{1} \approx 0.0009 r_{1}$. The calculated value is $\mathrm{k}$ $\sim L / \Delta r \approx 0.312$ and, at the same time, the empirical value of the von Karman constant is $\mathrm{k}=0.39 / 0.41$. Thus, the deviation in the calculated dependence from the empirical one is less than $25 \%$, which confirms the satisfactory agreement of dependence (22) for the critical point. It should be noted that the main studies [67-125] do not contain information on analytical solutions for the critical Taylor number as a function of the initial intensity and scale of perturbation. 


\section{Conclusions}

We presented the analytical Formula (13) for the critical Taylor number for the motion between rotating coaxial cylinders based on the theory of stochastic equations of continuum laws and the equivalence of measures between random and deterministic motions. The analytical Formula (22) for the critical point in the case of the motion between rotating coaxial cylinders is also derived. The result of the solution of Equation (13) shows a satisfactory correspondence with the obtained analytical dependence for the critical Taylor number to the experimental data. For validating the dependence for the critical point, the indirect calculated dependence of the von Karman constant was compared with the empirical value of this constant. The result of the comparison shows the satisfactory agreement between the calculated and empirical values of the von Karman constant.

Thus, the obtained analytical dependences can be used for validating both experimental and numerical studies of the onset of turbulence in a fluid flow between rotating cylinders, which is important for the practice of developing various technical devices such as hydraulic cylinders, as well as oil and water-cooling systems.

Funding: This work was supported by the program of increasing the competitive ability of the National Research Nuclear University MEPhI (agreement with the Ministry of Education and Science of the Russian Federation of 27 August 2013, Project No. 02.a03.21.0005).

Acknowledgments: The article is dedicated to Krivtsova Nelly Pavlovna.

Conflicts of Interest: The authors declare no conflict of interest.

\section{References}

1. Landau, L.D. Toward the problem of turbulence. Dokl. Akad. Nauk SSSR 1944, 44, 339-342.

2. Landau, L.D.; Lifshits, E.F. Fluid Mechanics; Perg. Press Oxford: London, UK, 1959.

3. Lorenz, E.N. Deterministic nonperiodic flow. J. Atmos. Sci. 1963, 20, 130-141. [CrossRef]

4. Feigenbaum, M. The transition to aperiodic behavior in turbulent sets. Commun. Math. Phys. 1980, 77, 65-86. [CrossRef]

5. Ruelle, D.; Takens, F. On the nature of turbulence. Commun. Math. Phys. 1971, 20, 167-192. [CrossRef]

6. Kolmogorov, A.N. Dissipation of energy in locally isotropic turbulence. Dokl. Akad. Nauk SSSR 1941, 32, 16-18.

7. Kolmogorov, A.N. A new metric invariant of transitive dynamic sets and automorphisms of the Lebesgue spaces. Dokl. Akad. Nauk SSSR 1958, 119, 861-864.

8. Kolmogorov, A.N. About the entropy per time unit as a metric invariant of automorphisms. Dokl. Akad. Nauk SSSR 1958, 124, 754-755.

9. Kolmogorov, A.N. Mathematical models of turbulent motion of an incompressible viscous fluid. Usp. Mat. Nauk 2004, 59, 5-10. [CrossRef]

10. Struminskii, V.V. Origination of turbulence. Dokl. Akad. Nauk SSSR 1989, 307, 564-567.

11. Klimontovich, Y.L. Problems of the statistical theory of open sets: Criteria of the relative degree ofthe ordering of states in the self-organization processes. Usp. Fiz. Nauk 1989, 158, 59-91. [CrossRef]

12. Samarskii, A.A.; Mazhukin, V.I.; Matus, P.P.; Mikhailik, I.A. Z/2 conservative schemes for the Korteweg-de Vries equations. Dokl. Akad. Nauk 1997, 357, 458-461.

13. Haller, G. Chaos near Resonance; Springer: Berlin, Germany, 1999. [CrossRef]

14. Orzag, S.A.; Kells, L.C. Transition to turbulence in plane Poiseuille and plane Couette flow. J. Fluid Mech. 1980, 96, 159-205. [CrossRef]

15. Ladyzhenskaya, O.A. On a dynamical system generated by Navier-Stokes equations. J. Sov. Math. 1975, 3, 458-479. [CrossRef]

16. Vishik, M.I.; Komech, A.I. Kolmogorov equations corresponding to a two-dimensional stochastic Navier-Stokes system. Tr. Mosk. Mat. Obs. 1983, 46, 3-43.

17. Packard, N.H.; Crutchfield, J.P.; Farmer, J.D.; Shaw, R.S. Geometry from a time series. Phys. Rev. Lett. 1980, 45, 712-715. [CrossRef]

18. Malraison, B.; Berge, P.; Dubois, M. Dimension of strange attractors: An experimental determination for the chaotic regime of two convective systems. J. Phys. Lett. 1983, 44, L897-L902. [CrossRef]

19. Procaccia, I.; Grassberger, P. Estimation of the Kolmogorov entropy from a chaotic signal. Phys. Rev. A 1983, 28, 2591-2593.

20. Constantin, P.; Foais, C.; Temam, R. On dimensions of the attractors in two-dimensional turbulence. Phys. D 1988, 30, 284-296. [CrossRef]

21. Grassberger, P.; Procaccia, I. Dimensions and entropies of strange attractors from a fluctuating dynamics approach. Phys. D Nonlinear Phenom. 1984, 13, 34-54. [CrossRef]

22. Rabinovich, M.I.; Reiman, A.M.; Sushchik, M.M.; Kozlov, V.V.; Ramazanov, M.P. Correlation dimension of the flow and spatial development of dynamic chaos in the boundary layer. JETP Lett. 1987, $13,987$. 
23. Brandstater, A.; Swift, J.; Swinney, H.L.; Wolf, A.; Farmer, D.J.; Jen, E.; Crutchfield, P.J. Low-dimensional chaos in hydrodynamic system. Phys. Rev. Lett. 1983, 51, 1442-1446. [CrossRef]

24. Sreenivasan, K.R. Fractals and multifractals in fluid turbulence. Ann. Rev. Fluid Mech. 1991, 23, 539-600. [CrossRef]

25. Priymak, V.G. Splitting dynamics of coherent structures in a transitional round-pipe flow. Dokl. Phys. 2013, 58, 457-465. [CrossRef]

26. Newton, P.K. The fate of random initial vorticity distributions for two-dimensional Euler equations on a sphere. J. Fluid Mech. 2016, 786, 1-4. [CrossRef]

27. Mayer, C.S.J.; von Terzi, D.A.; Fasel, H.F. Direct numerical simulation of investigation of complete transition to turbulence via oblique breakdown at Mach 3. J. Fluid Mech. 2011, 674, 5-42. [CrossRef]

28. Dmitrenko, A.V. Calculation of pressure pulsations for a turbulent heterogeneous medium. Dokl. Phys. 2007, 52, 384-387. [CrossRef]

29. Dmitrenko, A.V. Calculation of the boundary layer of a two-phase medium. High Temp. 2002, 40, 706-715. [CrossRef]

30. Dmitrenko, A.V. Heat and mass transfer and friction in injection to a supersonic region of the Laval nozzle. Heat Transf. Res. 2000, 31, 338-399. [CrossRef]

31. Dmitrenko, A.V. Film cooling in nozzles with large geometric expansion using method of integral relation and second moment closure model for turbulence. In Proceedings of the 33th AIAA/ASME/SAE/ASEE Joint Propulsion Conference and Exhibit, Seattle, WA, USA, 6-9 July 1997. AIAA Paper 97-2911. [CrossRef]

32. Dmitrenko, A.V. Heat and mass transfer in combustion chamber using a second-moment turbulence closure including an influence coefficient of the density fluctuation in film cooling conditions. In Proceedings of the 34th AIAA/ASME/SAE/ASEE Joint Propulsion Conference and Exhibit, Cleveland, OH, USA, 13-15 July 1998. AIAA Paper 98-3444. [CrossRef]

33. Dmitrenko, A.V. Nonselfsimilarity of a boundary-layer flow of a high-temperature gas in a Laval nozzle. Aviats. Tekh. 1993, 1, 39-42.

34. Dmitrenko, A.V. Computational investigations of a turbulent thermal boundary layer in the presence of external flow pulsations. In Proceedings of the 11th Conference on Young Scientists; Deposited at VINITI 08.08.86, No. 5698-B8; Moscow Physico-technical Institute: Moscow, Russia, 1986; Part 2; pp. 48-52.

35. Davidson, P.A. Turbulence; Oxford University Press: Oxford, UK, 2004.

36. Hinze, J.O. Turbulence, 2nd ed.; McGraw-Hill: New York, NY, USA, 1975.

37. Monin, A.S.; Yaglom, A.M. Statistical Fluid Mechanics; MIT Press: Cambridge, MA, USA, 1971.

38. Schlichting, H. Boundary-Layer Theory, 6th ed.; McGraw-Hill: New York, NY, USA, 1968.

39. Pope, S.B. Turbulent Flows; Cambridge University Press: Cambridge, UK, 2000. [CrossRef]

40. Dmitrenko, A.V. Fundamentals of heat and mass transfer and hydrodynamics of single-phase and two-phase media. In Criterialintegral Statistical Methods and Direct Numerical Simulation; Galleya Print: Moscow, Russia, 2008. Available online: http:/ / search.rsl.ru/ru/catalog/record/6633402 (accessed on 10 May 2020).

41. Dmitrenko, A.V. Equivalence of measures and stochastic equations for turbulent flows. Dokl. Phys. 2013, 58, 228-235. [CrossRef]

42. Dmitrenko, A.V. Regular Coupling between Deterministic (Laminar) and Random (Turbulent) Motions-Equivalence of Measures. 2013. Scientific Discovery Diploma No. 458, registration No. 583 of December 2. Available online: https://search.rsl.ru/ru/ record/01006633402 (accessed on 15 May 2020).

43. Dmitrenko, A.V. Some analytical results of the theory of equivalence measures and stochastic theory of turbulence for nonisothermal flows. Adv. Stud. Theor. Phys. 2014, 8, 1101-1111. [CrossRef]

44. Dmitrenko, A.V. Determination of critical Reynolds numbers for nonisothermalflows using stochastic theory of turbulence and equivalent measures. Heat Transf. Res. 2016, 47, 41-48. [CrossRef]

45. Dmitrenko, A.V. The theory of equivalence measures and stochastic theory of turbulence for non-isothermal flow on the flat plate. Int. J. Fluid Mech. Res. 2016, 43, 182-187. [CrossRef]

46. Dmitrenko, A.V. Analytical estimation of velocity and temperature fields in a circular tube on the basis of stochastic equations and equivalence of measures. J. Eng. Phys. Thermophys. 2015, 88, 1569-1576. [CrossRef]

47. Dmitrenko, A.V. An estimation of turbulent vector fields, spectral and correlation functions depending on initial turbulence based on stochastic equations. The Landau fractal equation. Int. J. Fluid Mech. Res. 2016, 43, 82-91. [CrossRef]

48. Dmitrenko, A.V. Stochastic equations for continuum and determination of hydraulic drag coefficients for smooth flat plate and smooth round tube with taking into account intensity and scale of turbulent flow. Contin. Mech. Thermodyn. 2017, 29, 1-9. [CrossRef]

49. Dmitrenko, A.V. Analytical determination of the heat transfer coefficient for gas, liquid and liquidmetal flows in the tube based on stochastic equations and equivalence of measures for continuum. Contin. Mech. Thermodyn. 2017, 29, 1197-1205. [CrossRef]

50. Dmitrenko, A.V. Determination of the coefficients of heat transfer and friction in supercritical-pressure nuclear reactors with account of the intensity and scale of flow turbulence on the basis of the theory of stochastic equations and equivalence of measures. J. Eng. Phys. Thermophys. 2017, 90, 1288-1294. [CrossRef]

51. Dmitrenko, A.V. Results of investigations of non-isothermal turbulent flows based on stochastic equations of the continuum and equivalence of measures. IOP Conf. Ser. J. Phys. Conf. Ser. 2018, 1009, 012017. [CrossRef]

52. Dmitrenko, A.V. The stochastic theory of the turbulence. IOP Conf. Ser. Mater. Sci. Eng. 2018, 468, 012021. [CrossRef]

53. Dmitrenko, A.V. Determination of the correlation dimension of an attractor in a pipe based on the theory of stochastic equations and equivalence of measures. J. Phys. Conf. Ser. 2019, 1705, 12015. [CrossRef] 
54. Dmitrenko, A.V. The construction of the portrait of the correlation dimension of an attractor in the boundary layer of Earth's atmosphere. J. Phys. Conf. Ser. 2019, 1301, 012006. [CrossRef]

55. Dmitrenko, A.V. The correlation dimension of an attractor determined on the base of the theory of equivalence of measures and stochastic equations for continuum. Contin. Mechan. Thermod. 2020, 32, 63-74. [CrossRef]

56. Dmitrenko, A.V. The possibility of using low-potential heat based on the organic Rankine cycle and determination of hydraulic characteristics of industrial units based on the theory of stochastic equations. JP J. Heat Mass Transf. 2020, 21, 125-132. [CrossRef]

57. Dmitrenko, A.V. The theoretical solution for the Reynolds analogy based on the stochastic theory of turbulence. JP J. Heat Mass Transf. 2019, 18, 463-476. [CrossRef]

58. Dmitrenko, A.V. Reynolds Analogy Based on the Theory of Stochastic Equations and Equivalence of Measures. J. Eng. Phys. 2021, 94, 186-193. [CrossRef]

59. Dmitrenko, A.V. Some aspects of the formation of the spectrum of atmospheric turbulence. JP J. Heat Mass Transf. 2020, 19, 201-208. [CrossRef]

60. Dmitrenko, A.V. Uncertainty relation in turbulent shear flow based on stochastic equations of the continuum and the equivalence of measures. Contin. Mech. Thermod. 2020, 32, 161-171. [CrossRef]

61. Dmitrenko, A.V. Formation of the turbulence spectrum in the inertial interval on the basis of the theory of stochastic equations and equivalence of measures. J. Eng. Phys. Thermophys. 2020, 93, 122-127. [CrossRef]

62. Dmitrenko, A.V. The Spectrum of the turbulence based on theory of stochastic equations and equivalence of measures. J. Phys. Conf. Ser. 2020, 1705, 012021. [CrossRef]

63. Dmitrenko, A.V. Theoretical solutions for spectral function of the turbulent medium based on the stochastic equations and equivalence of measures. Contin. Mech. Thermod. 2021, 33, 603-610. [CrossRef]

64. Dmitrenko, A.V. Determination of critical Reynolds number in the jet based on the theory of stochastic equations and equivalence of measures. J. Phys. Conf. Ser. 2020, 1705, 012015. [CrossRef]

65. Dmitrenko, A.V. Determination of Critical Reynolds Number for the Flow Near a Rotating Disk on the Basis of the Theory of Stochastic Equations and Equivalence of Measures. Fluids 2021, 6, 5. [CrossRef]

66. Taylor, G.I. Stability of a viscous liquid contained between two rotating cylinders. Proc. Roy. Soc. 1935, A151, 494-512.

67. Taylor, G.I. Internal waves and turbulence in affluid of variable density. Rapp. Proc. Verb. Cons. Internat. 1931, 132, 35-42.

68. Taylor, G.I. Effects of variation in density on the stability of superposed streams of fluid. Proc. Roy. Soc. 1931, A132, 499-523.

69. Tetervin, N. Charts and tables for estimating the stability of the compressible laminar boundary layer with heat transfer and arbitrary pressure gradient. NASA MEMO 1959, 5, 1-48.

70. Wendt, F. Turbulente Stromung zwischenlzwei rotierenden koaxialen Zylindern. Ingenieur-Archiv 1933, 4, 577-595. [CrossRef]

71. Synge, J.L. On the stability of a viscous liquid between rotating coaxial cylinders. Proc. Roy. Soc. 1938, A167, 250-256.

72. Veite, W. Stabilität und Verzweigung stationärer lösungen der Navier-Stokesschen gleichungen beim Taylor problem. Arch. Rat. Mech. Anal. 1966, 22, 1-14.

73. Koschmieder, E.L. Bénard Cells and Taylor Vortices; Cambridge University Press: Cambridge, UK, 1993; ISBN 0-521-40204-2.

74. Andereck, C.D.; Liu, S.S.; Swinney, H.L. Flow regimes in a circular Couette system with independently rotating cylinders. J. Fluid Mech. 1986, 164, 155-183. [CrossRef]

75. Kao, K.-H.; Chow, C.-Y. Linear stability of compressible Taylor-Couette flow. Phys. Fluids A Fluid Dyn. 1992, 4, 984-996. [CrossRef]

76. Koschmieder, E.L. Transition from laminar to turbulent Taylor vortex flow. In Laminar-Turbulent Transition Symp; Springer: Berlin, Germany, 1980; pp. 396-404.

77. Kroner, K.H.; Nissinen, V. Dynamic filtration of microbial suspensions using an axially rotating filter. J. Membr. Sei. 1988, 36, 85100. [CrossRef]

78. Chandrasekhak, S. Hydrodynamic and Hydromagnetic Stability; Clarendon Press: Oxford, UK, 1961; 852p.

79. Krueger, E.R.; Gross, A.; Di Prima, R.C. On the relative importance of Taylor-vortex and non-axisymmetric modes in flow between rotating cylinders. J. Fluid Mech. 1966, 24, 521-538. [CrossRef]

80. Donnelly, R.J. Experiments on the stability of viscous flow between rotating cylinders. Proc. R. Soc. Ser. A 1958, 246, 312-325.

81. Donnelly, R.J.; Simon, N.J. An empirical torque relation for supercritical flow between rotating cylinders. J. Fluid Mech. 1960, 7 , 401-418. [CrossRef]

82. Di Prima, R.C. Stability of nonrotating symmetric disturbances for viscous flow between rotating cylinders. Phys. Fluids 1961, 4 , 751-755. [CrossRef]

83. Davey, A. The growth of Taylor vortices in flow between rotating cylinders. J. Fluid Mech. 1962, 14, 336-368. [CrossRef]

84. Davey, A.; Di Prima, R.C.; Stuart, J.T. On the instability of Taylor vortices. J. Fluid Mech. 1968, 31, 17-52. [CrossRef]

85. Lueptow, R.M.; Hajiloo, A. Flow in a rotating membrane plasma separator. Trans. Am. Soc. Artif. Intern. Organs 1995, 41, 182-188. [CrossRef]

86. Feigenbaum, M.I. Universal behaviour in nonlinear systems. Los Alamos Sei. 1980, 1, 4-27.

87. Burkhalter, J.E.; Koschmieder, E.L. Steady supercritical Taylor vortices after sudden starts. Phys. Fluids 1974, 17, 1929-1935. [CrossRef]

88. Lueptow, R.M.; Min, K. Circular Couette flow with pressufe-driven axial flow and a porous inner cylinder. Exp. Fluids 1994, 17, 190-197. 
89. Lueptow, R.M.; Min, K. Hydrodynamic stability of viscous flow between rotating porous cylinders with radial flow. Phys. Fluids 1994, 6, 144-151.

90. Mallock, A. Determination of the viscosity of water. Proc. Roy. Soc. 1888, A45, 126-132.

91. Meyer-Spasche, R.; Keller, H.B. Computation of the axisymmetric flow between rotating cylinders. J. Comput. Phys. 1980, 35, 100109. [CrossRef]

92. Ohashi, K.; Tashiro, K.; Kushiya, F.; Matsumoto, T.; Yoshida, S.; Endo, M.; Horio, T.; Ozawa, K.; Sakai, K. Rotation-induced Taylor vortex enhances filtrate flux in plasma separation. ASAIO Trans. 1988, 34, 300-307. [PubMed]

93. Wimmer, M. An experimental investigation of Taylor vortex flow between conical cylinders. J. Fluid Mech. 1995, $292,205-227$. [CrossRef]

94. Wimmer, M. Experiments on a viscous fluid flow between concentric rotating spheres. J. Fluid Mech. 1976, 78, 317-335. [CrossRef]

95. Ovchinnikova, S.N.; Yudovich, V.I. Resonances in the intersections of bifurcation in the Couette-Taylor problem. In Patterns and Waves; Abramian, A., Vakulenko, S., Volpert, V., Eds.; 2003; p. 5577. Available online: https://www.dissercat.com/content/ dvizheniya-zhidkosti-mezhdu-vrashchayushchimisya-pronitsaemymi-tsilindrami (accessed on 15 May 2020).

96. Rayleigh, L. On Convention Currents in a Horizontal Layer of Fluid When the Higher Temperature Is on the under Side; Sei. Papers; Cambridge University Press: Cambridge, UK, 1916; Volume 6, pp. 529-546.

97. Shapakidze, L. On the bifurcation of flows of a heat-conducting fluid between two rotating permeable cylinders. Georgian Math. J. 1997, 4, 567-578. [CrossRef]

98. Shapakidze, L. On the stability of flows between two rotating permeable cylinders. Proc. Int. Conf. Appl. Mech. 1989, 1, 450454.

99. Swinney, H.L.; Gollub, J.P. Transition to turbulence. Phys. Today 1978, 31, 41-49. [CrossRef]

100. Wronski, S.; Molga, E.; Rudniak, L. Dynamic filtration in biotechnology. Bioprocess Eng. 1989, 4, 99-104. [CrossRef]

101. Yahata, H. Dynamics of the Taylor vortices above higher instability points. Progr. Theor. Phys. 1978, 59, 1755-1756. [CrossRef]

102. Yahata, H. Temporal development of the Taylor vortices in a rotating fluid. Ill. Progr. Theor. Phys. 1980, 64, 782-793. [CrossRef]

103. Yahata, H. Temporal development of the Taylor vortices in a rotating fluid. 1. Progr. Theor. Phys. 1981, 66, 879-891. [CrossRef]

104. Yahata, H. Temporal development of the Taylor vortices in a rotating fluid. 274. Progr. Theor. Phys. 1983, 69, 396-402. [CrossRef]

105. Schwüle, J.A.; Mitra, D.; Lueptow, R.M. Design parameters for rotating filtration. J. Membrane Sei. 2002, 204, 53-65. [CrossRef]

106. Schwüle, J.A.; Mitra, D.; Lueptow, R.M. Anti-fouling mechanism in rotating filtration. In Proceedings of the 12th International Couette-Taylor Workshop, Evanston, IL, USA, 6-8 September 2001. Session 2D.

107. Serre, E.; Sprague, M.A.; Lueptow, R.M. Stability of Taylor-Couette flow in a finite-length cavity with radial throughflow. Phys. Fluids. 2008, 20, 034106-1-034106-10. [CrossRef]

108. Shah, T.N.; Yoon, Y.; Pederson, C.L.; Lueptow, R.M. Rotating reverse osmosis and spiral wound reverse osmosis filtration: A comparison. J. Membrane Sei. 2006, 285, 353-361. [CrossRef]

109. Kolesov, V.; Shapakidze, L. On oscillatory modes in viscous incompressible liquid flows between two counter-rotating permeable cylinders. Trends App. Math. Mech. Boca Raton Chapman Hall CRC. 2000, 106, 221-227.

110. Kolesov, V.; Shapakidze, L. On transitions near the intersection point of bifurcations in the flow between two rotating permeable cylinders. Proc. A. Razmadze Math. Inst. 2000, 122, 79-91.

111. Jain, N.C.; Bansal, J.L. On the flow of a viscous incompressible fluid between two coaxial rotating porous cylinders. Proc. Indian. Acad. Sei. Math. Sei. 1973, 78, 187-201. [CrossRef]

112. Johnson, C.; Lueptow, R.M. Hydrodynamic stability of flow between rotating porous cylinders with radial and axial flow. Phys. Fluids 1997, 9, 3687-3696. [CrossRef]

113. Fenstermacher, P.R.; Swinney, H.L.; Benson, S.V.; Gollub, J.P. Bifurcations to periodic, quasiperiodic, and chaotic regimes in rotating and convecting fluids. Ann. N. Y. Acad. Sei. 1979, 316, 652-666. [CrossRef]

114. Fenstermacher, P.R.; Swinney, H.L.; Gollub, J.P. Dynamical instabilities and the transition to chaotic Taylor vortex flow. J. Fluid Mech. 1979, 94, 103-128. [CrossRef]

115. Frank, G.; Meyer-Spasche, R. Computation of transitions in Taylor vortex flows. Z. Angev. Math. Und. Phys. 1981, 32, 710-720. [CrossRef]

116. Goharzadeh, A.; Mutabazi, I. Experimental characterization of intermittency regimes in the Couette-Taylor System. Eur. Phys. J.B. 2001, 19, 157-162. [CrossRef]

117. Lee, S.; Lueptow, R.M. Rotating membrane filtration and rotating reverse osmosis. J. Chem. Eng. Japan 2004, 37, 471-482. [CrossRef]

118. Chang, M.-H. Hydrodynamic stability of Taylor-Dean flow between rotating porous cylinders with radial flow. Phys. Fluids 2003, 15, 1178-1188. [CrossRef]

119. Serre, E.; Sprague, M.; Bontoux, P.; Lueptow, R.M. The effect of radial through-flow on the stability of Taylor-Couette flow. In Proceedings of the 15th Intern. Couette-Taylor Workshop, Le Havre, France, 9-12 July 2007.

120. Richard, D. Sandberg Governing Equations for a New Navier-Stokes Solver in General Cylindrical Coordinates; Report No. AFM-07/07; University of Southampton: Southampton, UK, 2007. Available online: http://eprints.soton.ac.uk/49523 (accessed on 15 May 2020).

121. Dou, H.S. Mechanism of flow instability and transition to turbulent. Int. J. Non-Linear Mech. 2006, 41, 512-517. [CrossRef]

122. Dou, H.S.; Khoo, B.C. Investigation of turbulent transition in plane Couette flows using energy gradient method. Adv. Appl. Math. Mech. J. 2011, 3, 165-180. [CrossRef] 
123. Leclercq, C.; Nguyen, F.; Kerswell, R.R. Connections between centrifugal, stratorotational and radiative instabilities in viscous Taylor-Couette flow. Phys. Rev. E 2016, 94, 43-103. [CrossRef]

124. Ilin, K.; Morgulis, A. Instability of an inviscid flow between porous cylinders with radial flow. J. Fluid Mech. 2013, 730, $364-378$. [CrossRef]

125. Ilin, K.; Morgulis, A. On the stability of the Couette-Taylor flow between rotating porous cylinders with radial flow. Eur. J. Mech. B/Fluids 2020, 80, 174-186. [CrossRef] 